\title{
The new Japanese Classification of Gastric Carcinoma: Revised explanation of "Response assessment of chemotherapy and radiotherapy for gastric carcinoma"
}

\author{
Minoru Kurihara ${ }^{1}$ and Takashi Aiko ${ }^{2}$ for the General Rules' Committee of the Japanese Gastric Cancer \\ Association* \\ ${ }^{1}$ Department of Gastroenterology, Toyosu Hospital, 4-1-18 Toyosu, Koto-ku, Tokyo 135-8577 Japan \\ ${ }^{2}$ First Department of Surgery, Kagoshima University, Kagoshima, Japan
}

Key words Response assessment - Chemo/radiotherapy · Primary focus $\cdot$ Gastric cancer

\section{Background}

The Response assessment of chemotherapy for gastric carcinoma (the Japanese evaluation criteria) was first introduced in the 11th Japanese edition [1] of the General rules (GR) for gastric cancer study of the Japanese Research Society for Gastric Cancer in 1985.

The principles of the World Health Organization (WHO) criteria were respected in this new method. Two important elements were: (1) using X-ray and endoscopic findings to assess gastric lesions, and (2) a new methodology to evalute diffusely infiltrating tumors.

Primary lesions were classified as: (a) measurable, (b) evaluable but not measurable (lesions extending to more than half the circumference of the lumen of the organ that could not be measured, and lesions with unclear boundaries that were examined carefully and comparatively, not only by an upper gastrointestinal Xray examination but also by endoscopic examination), and (c) diffusely infiltrating lesions, represented by linitis plastica. Findings of regression, defined as a $50 \%$ reduction in tumor size in (a) lesions and also an around $50 \%$ reduction in the ulcerous area and a flattening of surrounding bank in (b) lesions, were designated as

Offprint requests to: $\mathrm{M}$. Kurihara

Received: January 15, 2001 / Accepted: February 15, 2001

* Committee members

Tetsuro Kubota, Keio University, Tokyo; Wasaburo Koizumi, Kitazato University, Sagamihara; Tsuneo Sasaki, Tokyo Metropolitan Komagome Hospital, Tokyo; Hiroto Matsue, National Cancer Center Hospital, Tokyo; Shigeaki Yoshida, National Cancer Center Hospital East, Kashiwa. partical response (PR), while in (c) lesions, more than $50 \%$ (changed from $100 \%$ in the 12 th edition [2]) enlargement in the affected area was designated as PR (Fig. 1).

In 1991, Kurihara and the Gastric Cancer Study Subgroup (18 institutions throughout Japan) of the Japan Clinical Oncology Group (JCOG) clarified 386 inoperable primary lesions treated by various anticancer chemotherapies, and classified 80 measurable lesions (a), 156 evaluable but not measurable lesions (b), and 150 diffusely infiltrating lesions (c), with response rates of $15 \%, 18 \%$, and $2.7 \%$, respectively, for a strictly extramural review [3].

Ohtsu and the JCOG analyzed 226 patients with advanced gastric cancer who had no prior treatment [4]. Antitumor responses were evaluated by the Japanese Evaluation Criteria. Of the 226 patients, 192 (85\%) presented with primary cancers. Only 57 of these 192 patients had roentgenographically measurable tumors. The response rate of the primary cancers was significantly lower than that of measurable metastatic lesions $(P<0.01)$, as shown in Table 1 .

Forty-five responders with both primary and metastatic tumors were divided into 31 responders and 14 nonresponders, for reference to primary cancers. The 31 responders in the primary cancer group survived significantly longer $(P<0.05)$ than did the 14 nonresponders (Fig. 2), even though there was no significant difference in patient background between the two groups (Table 2). This difference in these survival rates became even more remarkable after 1 year. Not only Ohtsu et al. [4] but also other researchers revealed that the response rate of c-lesions was significantly lower than those of a- and b-lesions. For instance, randomized controlled trials of two regimens, Tegafur (Futrafur) plus mitomycin C (MMC) (FTM therapy) and Combined Tegafur and Uracil (UFT) + MMC (UFTM therapy) showed response rates of $11.5 \%$ and $40.7 \%$ in a-lesions (total response, $26.4 \%$ ), $9.8 \%$ and 


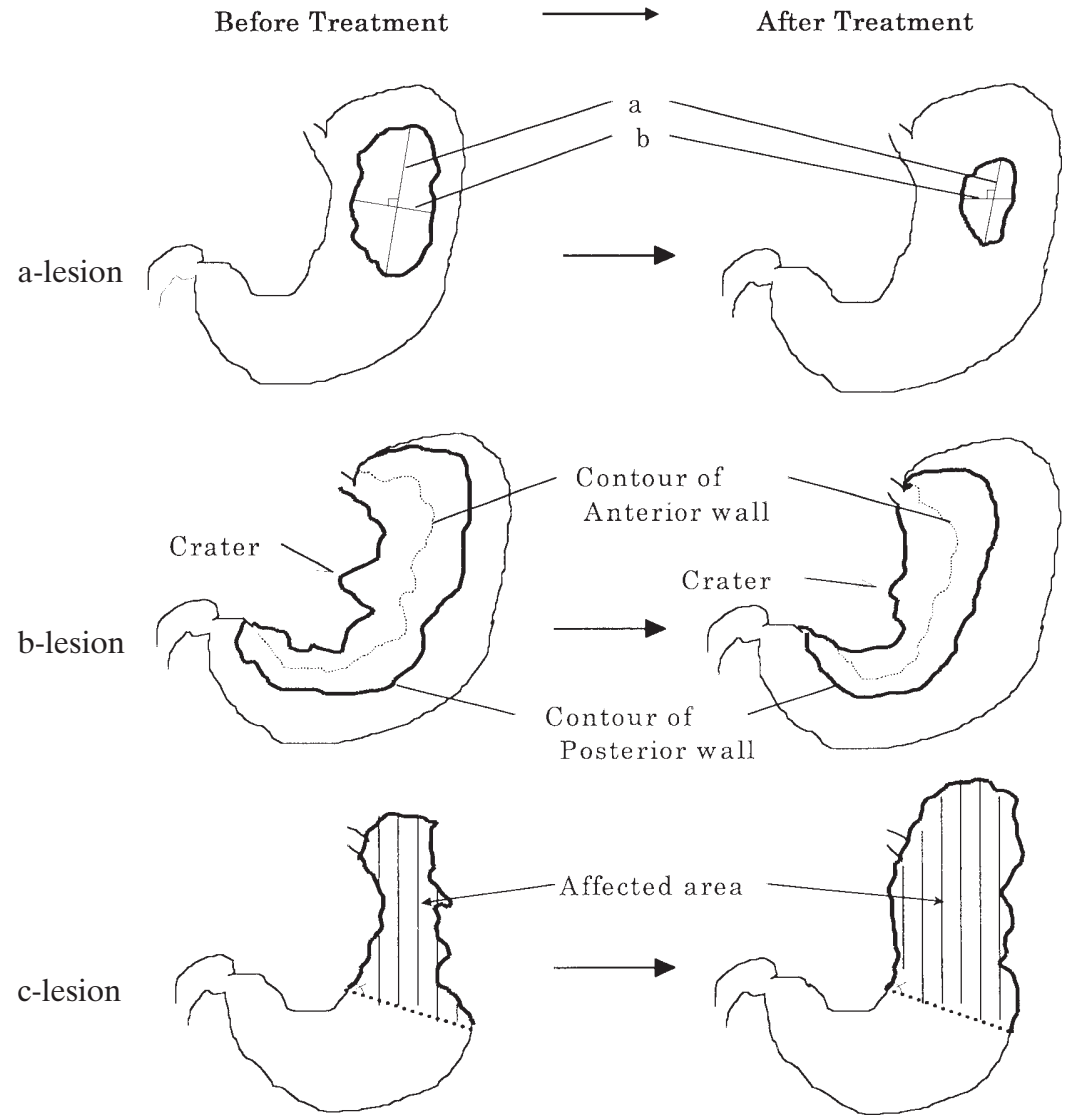

Fig. 1. Partial response (PR) according to the Japanese Response Criteria [10] for primary gastric cancer lesions. Measurable lesion ( $a$ lesion): more than $50 \%$ decrease of total tumor size on X-ray and/or endoscopic examinations for more than 4 weeks. Evaluable but not measurable lesion (b-lesion): findings clearly differ from pretreatment examinations, e.g., showing marked regression and flattening of elevated or ulcerative lesion (estimated decrease of more than $50 \%$ ) in regard to macroscopic change on $\mathrm{X}$-ray and/or endoscopic examination for more than 4 weeks. Diffusely infiltrating lesion $(c$ lesion): enlargement of more than $50 \%$ of the gastric lumen of the affected region (striped area) on X-ray examination for more than 4 weeks

Table 1. Response of primary and secondary cancers to chemotherapy in patients with gastric cancer

\begin{tabular}{lcr}
\hline Variable & $\begin{array}{c}\text { Number of } \\
\text { patients }(\%)\end{array}$ & $\begin{array}{c}\text { Response } \\
\text { rate }(\%)\end{array}$ \\
\hline Primary cancer & & \\
a-lesion & $57(30)$ & $15 / 57(26)$ \\
b-lesion & $76(40)$ & $24 / 76(32)$ \\
c-lesion & $59(31)$ & $1 / 59(2)$ \\
Total & $192(100)$ & $40 / 192(21)$ \\
Metastatic cancer & & \\
Liver & $97(49)$ & $34 / 97(35)$ \\
Abdominal lymph node & $64(32)$ & $24 / 64(38)$ \\
Cervical lymph node & $24(12)$ & $11 / 24(46)$ \\
Other & $13(7)$ & $5 / 13(38)$ \\
Overall & $198(100)$ & $74 / 198(37)$
\end{tabular}

See text for explanation of "a", "b" and "c" lesions

$25 \%$ in b-lesions (total response, $16.4 \%$ ), and $3.2 \%$ and $8.3 \%$ in c-lesions (total 5.5\%), respectively, although median survival time (MST) was the same (180 days) with both regimens. In a total of 169 patients, MST was 282 days in those with a-lesions, 161 days in those with b-lesions, and 189 days in those with c-lesions [5].

In a pilot chemotherapy study with Doxifluridine (5'DFUR) plus cisplatin (CDDP) in 64 patients, however, response rates were $47.1 \%$ in a-lesions, $37 \%$ in blesions, and 5\% in c-lesions. MST was 273 days in those

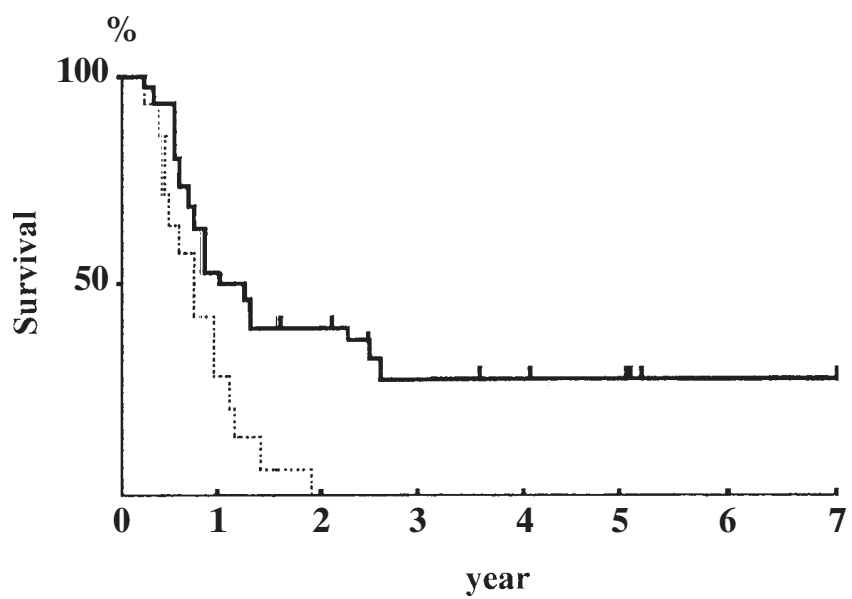

Fig. 2. Survival times of overall responders, according to the response to chemotherapy, in 45 patients with both primary gastric cancer and metastatic lesions. Solid line represents the survival time of patients with chemotherapeutic response to primary cancers $(n=31)$. Dotted line represents the survival time of patients with chemotherapeutic nonresponse to primary cancers $(n=14)$

with a-lesions, 267 days in those with b-lesions, and 287.5 days in those with c-lesions [5].

In 1993, Kurihara et al. [5] reported that, in 8 "minor response (MR)" patients (enlargement of affected area 
Table 2. Characteristics of gastric cancer patients with primary and metastatic tumors who were overall responders; in relation to the primary cancer

\begin{tabular}{lccr}
\hline Variable & $\begin{array}{c}\text { Responder } \\
\text { in primary cancer }\end{array}$ & $\begin{array}{c}\text { Nonresponder } \\
\text { in primary cancer }\end{array}$ & Total \\
\hline Number of patients & 31 & 14 & 45 \\
Performance status & 27 & 13 & 40 \\
$\quad 0,1$ & 4 & 1 & 5 \\
$\quad 2$ & 16 & 7 & 23 \\
Histological type & 15 & 7 & 22 \\
$\quad$ Intestinal & 20 & 8 & 28 \\
$\quad$ Diffuse & 15 & 9 & 6 \\
Metastastic site & 4 & 2 & 38 \\
$\quad$ Liver & & & 7 \\
$\quad$ Lymph node & 26 & 2 & \\
$\quad$ Other & 5 & & \\
Chemotherapy regimen type & & & \\
$\quad$ With cisplatin & & & \\
$\quad$ Without cisplatin & & & \\
\hline
\end{tabular}

Numbers of patients are shown for all variables except for metastatic site (some patients had metastasis at more than one site)

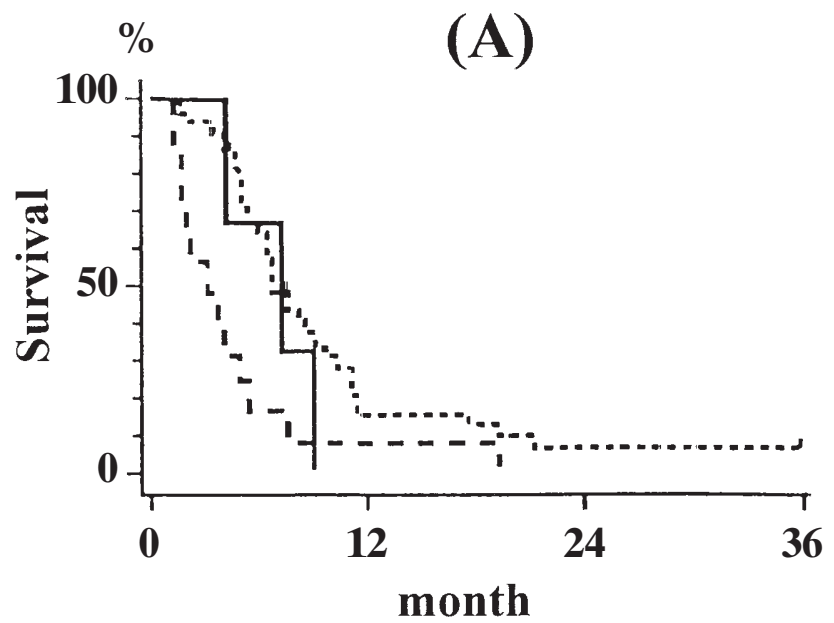

Fig. 3A,B. Survival times for chemotherapeutic responses to Tegafur plus mitomycin C (MMC) or Combined Tegafur and Uracil (UFT) plus MMC in c-lesion gastric cancer patients in whom partial response (PR) was defined as in references [1] and [2]. In c-lesions, in reference [1] partial response $(P R$; continuous line) to chemotherapy was defined as $100 \%$ enlargement of the affected area for more than 4 weeks, and no change (NC; dotted line) was defined as less than $100 \%$ enlargement or nonenlargement for more than 4 weeks, on X-

$\geqq 50 \%,<100 \%$ ) with c-lesions treated with FTM and UFTM, this chemotherapy, study resulted in a prolonged MST of 450.5 days, longer than the 411-day MST in $13 \mathrm{PR}$ patients with a-lesions and the 295.5-day MST in $10 \mathrm{PR}$ patients with b-lesions. Survival times in patients with PR according to the criteria in [1] (enlargement of affected area $\geqq 100 \%$ ) were compared with PR those with according to the new criteria (enlargement of

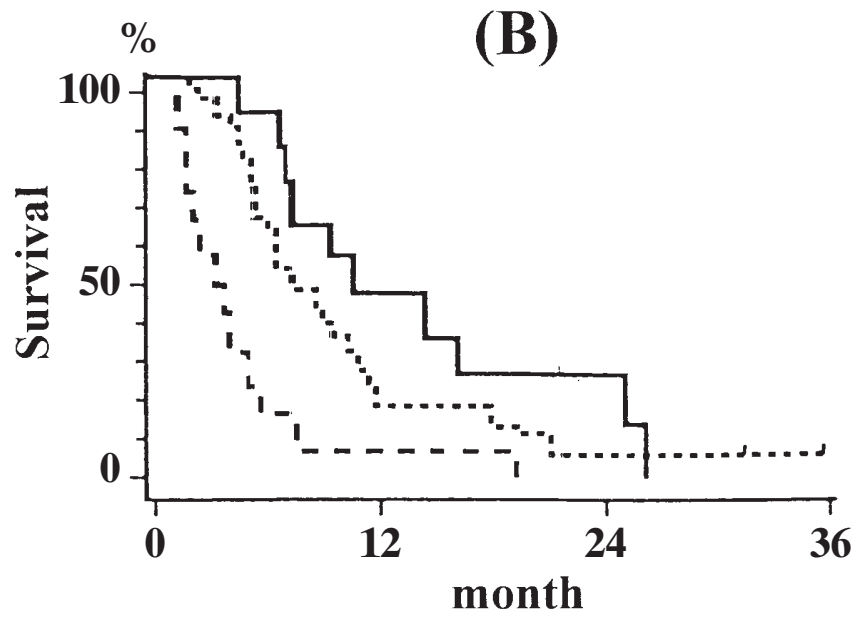

ray examination (A). In c-lesions, according to reference [2] PR was defined as more than $50 \%$ (but less then 100\%) enlargement of the affected area for more than 4 weeks, and $\mathrm{NC}$ was defined as less than $50 \%$ enlargement or nonenlargement for more than 4 weeks, on X-ray examination (B). According to both references [1] and [2], progressive disease $(P D ;$ dashed line $)$ in c-lesion gastric cancer was defined as a decrease of the affected area on X-ray examination, or the appearance of new lesions

affected area $\geqq 50 \%$ ) in 55 c-lesion patients (Fig. 3). MST based on the $\geqq 50 \%$ increase of the affected area in c-lesions was 314 days for PR and 211 days for NC, showing a significant difference, indicating a significant correlation between chemotherapeutic efficacy and survival time in c-lesion patients [5]. Therefore, on the basis of previous study findings, in the 12th edition of the GR [2], the PR definition for c-lesions was revised. 
Recently, Koizumi et al. [6] reported 48 responders $(53.3 \%)$ and 42 nonresponders $(46.7 \%)$ in a total of 90 patients with inoperable gastric primary tumors treated by various chemotherapies, consisting of fluorinated pyrimidine (i.v. or p.o.) and CDDP, using the Japanese criteria. MSTs were: 9.4 months overall, 12.6 months in responders (14.2 months in 23 a-lesion patients, 11.5 months in 12 b-lesion patients, and 10.5 months in 13 c-lesion patients) and 7.8 months in the nonresponders (11.4 months in 4 a-lesion patients, 7.4 months in 19 b-lesion patients, and 7.2 months in 19 c-lesion patients). It was pointed out that, in inoperable gastric cancer patients without measurable metastatic lesions (for instance, those in liver, lung, and lymph nodes) only 51 of the total of 90 patients could be evaluated by the WHO criteria [7], which are the most commonly used in the United States and Europe.

Kurihara et al. [8] reported results similar, to those of Koizumi et al. [6], with 41 responders in a total of 118 patients treated with $5^{\prime}$-DFUR plus CDDP, by the Japanese Criteria (response rate, $34.7 \%$ ); MSTs were: 268 days overall, 425 days in the 41 responders, and 188 days in the 77 nonresponders. However, according to the WHO criteria, and especially in regard to the Response Evaluation Criteria in Solid Tumors RECIST [9], 35 of the 118 patients above, who had inoperable primary gastric tumors invading adjacent organs, or cancerous peritonitis, not detectable by computed tomography (CT) or magnetic resonance imaging (MRI), could not be enrolled in clinical trials.

\section{Introduction}

The first English edition of the Japanese classification of gastric carcinoma was published in 1995 [10], based on the 12th Japanese edition [2] of The general rules (GR), for gastric cancer study of the Japanese Research Society for Gastric Cancer, with full illustrations and detailed descriptions. To coincide with the establishment of the Japanese Gastric Cancer Association (JGCA) in 1998, the 13th edition of the GR [11] and the 2nd English edition of the JGCA classification, except for "Part IV: response assessment of chemotherapy for gastric carcinoma" [12], were published after correction and revision of the 12th edition of the GR and the 1st English edition, respectively.

In this article, "Part IV: response assessment of chemo/radiotherapy for gastric carcinoma," which has been translated from the 13th Japanese edition, is introduced. As already described in the 1st English edition in Japan, inoperable gastric tumors, as well as metastatic lesions treated by chemo/radiotherapy, have been assessed. For the assessment of primary gastric lesions, radiological and endoscopic findings are used at 4-week intervals. Primary lesions were classified as follows: (a) measurable, (b) evaluable but not measurable, and (c) diffusely infiltrating lesions (Fig. 1). Since 1993 (12th edition [2]), these assessment methods have been widely accepted by specialists after being discussed at our meetings throughout Japan.

On overall evaluations, the treated patients assessed by the Japanese criteria had a higher survival rate (Fig. 2), suggesting that the antitumor effect was closely related to the survival time [4]. With new regimens, similar results were observed [5,6], as described in "Background." Therefore, the response assessments for primary gastric lesions are, in general, the same as those in the 1st English edition. However, as some revisions, considered to be reasonable, were those included, they are summarized below.

\section{Summary of changes}

1. The previous English version of the Japanese classification of gastric carcinoma [10] described the assessment of primary gastric lesions, using X-ray and endoscopic findings; the lesions were classified as: (a) measurable lesions, (b) evaluable but not measurable, and (c) diffusely infiltrating. The present version [11] revised the assessment method in detail.

2. Patient status at the start of therapy: (1) Performance status (PS) according to the "WHO criteria," (2) concurrent disease(s) that may influence the outcome of chemotherapy or radiation therapy, (3) description of lesions: presence of previous treatment and recurrence, as well as "the stage" [12].

3. Therapy description: details of chemotherapy and radiation therapy have to be described.

4. Clinical efficacy, maximum change in pain, anorexia, nausea-vomiting, changes in body weight, PS, serum tumor marker levels, and quality of life (QOL) score on a specified questionnaire [13-15].

5. The determination of overall response includes not only eligible cases and complete cases but also the "Full analysis set [16]," and there have to be population parameters.

6. When evaluating responses in patients with carcinomas, only CR should be included in the calculations. Cytodiagnosis is not necessary in the evaluation of the response; however, when possible, the results should be described separately.

7. Duration of survival, population parameters, and overall response rate should be described.

8. The 1- to 5-year cumulative survival rates in the treated population should be calculated by the Kaplan-Meier method or the life-table method.

9. Adverse drug reactions should describe the toxicity, severity, date of onset, and duration, according to 
the Revised NCI-Common Toxicity Criteria (CTC) version 2.0, Jan. 30, 1998.

10. Factors related to tumor response should be stratified to evaluate the response.

There are no international guidelines for the assessment of response for primary gastrointestinal lesions. The efficacy assessment is often done only for metastatic lesions. The possibility that primary lesions were not checked despite having a response categorized as PD cannot be ruled out. However, in the RECIST criteria, gastric primary lesions are dealt with as "nontarget lesions" [9].

The Japanese criteria are useful for correlating the antitumor effects achieved by chemotherapies in the primary tumors; however, further studies are warranted, both in Japan and throughout the world.

Acknowledgments The Association wishes to thanks Dr. Jaffer A Ajani, University of Texas MD Anderson Cancer Center, Texas, USA; and Dr. Scott A Hundahl, The Queen's Medical Center, Hawaii, USA, for their valuable assistance. The authors express sincere thanks to Mr. Robert E. Brandt for English editing and apt suggestions.

\section{References}

1. Japanese Research Society for Gastric Cancer. The general rules for gastric cancer study (in Japanese). 11th ed. Tokyo: Kanehara; 1985.

2. Japanese Research Society for Gastric Cancer. The general rules for gastric cancer study (in Japanese). 12th ed. Tokyo: Kanehara; 1993.

3. Kurihara M, Izumi T, Ito T. Evaluation of the effect of chemotherapy in gastric cancer patients with an intact primary tumor according to the criteria of the Japanese Research Society for Gastric Cancer. J Jpn Soc Cancer Ther 1991;26:644-54.

4. Ohtsu A, the Japan Clinical Oncology Group. Response of the primary lesion in gastric cancer to chemotherapeutic trials. Int $\mathbf{J}$ Clin Oncol 1998;3:3-6.
5. Kurihara M, Matsukawa M. Evaluation of efficacy of chemotherapy/radiation therapy in the treatment of inoperative gastric cancer with primary foci. In: Takahashi $\mathrm{T}$, editor. Recent advances in management of digestive cancers. Tokyo Berlin Heidelberg New York: Springer-Verlag; 1993. pp 132-7.

6. Koizumi W, Kurihara M, Tanabe S. the Advanced Gastric Cancer Cooperative Study Group. Advantage of Japanese response criteria for estimating the survival of patients with primary gastric cancer. Gastric Cancer 1999;2:14-19.

7. World Health Organization. WHO Handbook for reporting results of cancer treatment. WHO offset publication no. 48. Geneva, Switzerland: World Health Organization; 1979.

8. Kurihara M, Koizumi M, Sato A. Response assessment of chemotherapy for gastric cancer (in Japanese). Jpn J Cancer Clin 1999; 45:751-8.

9. Therasse P, Arbuck SG, Eisenhauer EA, Wanders J, Kaplan RS, Rubinstein L, et al. New guidelines to evaluate the response to treatment in solid tumors. European Organization for Research and Treatment of Cancer, National Cancer Institute of the United States, National Cancer Institute of Canada. J Natl Cancer Inst 2000;92:205-16.

10. Japanese Research Society for Gastric Cancer. Japanese classification of gastric carcinoma, 1st English ed. Tokyo: Kanehara; 1995.

11. Japanese Research Society for Gastric Cancer: The general rules for gastric cancer study (in Japanese). 13th ed. Tokyo: Kanehara; 1999.

12. Japanese Research Society for Gastric Cancer. Japanese classification of gastric carcinoma, 2nd English ed. Gastric Cancer 1998;1:18-19.

13. Kurihara M, Shimizu H, Tsuboi K, Kobayashi K, Murakami M, Eguchi K, Shimozuma K. Development of quality of life questionnaire in Japan: quality of life assessment of cancer patients receiving chemotherapy. Psychooncology. 1999;8:355-63.

14. Aaronson NK, Ahmedzai S, Bergman B, Bullinger M, Cull A, Duez NJ, et al. The European Organization for Research and Treatment of Cancer QLQ-C30: a quality-of-life instrument for use in international clinical trials in oncology. J Natl Cancer Inst 1993;85:365-76.

15. Cella DF, Tulsky DS, Gray G, Sarafian B, Linn E, Bonomi A, et al. The functional assessment of cancer therapy scale: development and validation of the general measure. J Clin Oncol 1993;11:570-9.

16. International conference on harmonisation; guidance on statistical principles for clinical trials; availability-FDA. Notice Fed Regist 1998;63:49583-98. 